
on Recombination in Semiconductors, University of Southampton, U.K. 30 August - 1 September 1978

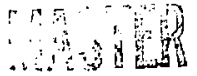

\title{
LIFETIME AND RADIATIVE EFFICIENCY VS DENSITY IN THE STRAIN-CONFINED ELECTRON-HOLE LIQUID IN Ge
}

Susan M. Kelso and John E. Furneaux

Apr11 1978

Prepared for the U. S. Department of Euergy under Contract $\mathrm{W}-7405-\mathrm{ENG}-48$ 
LIFETIME AND RADIATIVE EFFICIENCY VS DENSITY IN THE STRAIN-CONFINED ELECTRON-HOLE LIQUID IN Ge

Susan M. Kelso and John E. Furneaux

Physics Department

University of California

Berkeley, California 94720 USA

and

\author{
Materials and Molecular Research Division \\ Lawrence Berkeley Laboratory \\ Berkeley, California 94720 USA
}

\title{
ABSTRACT
}

We have performed two experiments on the electron-hole liquid (EHL) In Inlomogeneously stressed Ge in order to obtaln information on the lifetime and radiative efficiency as a function of e-h palr density in the strain-confined electron-hole liquid (SCEHL). Our data can only be explained if a density-independent recombination mechanistn is the most Important decay process. We show that our result is consistent with other experiments indicating that such a mechanism has a negligible effect on the recombination time in unstressed Ge. 
The electron-hole 1iquid (EHL) In semiconductors has been the subfect of much experimental and theoretical study over the last decade. (1) This Fermi fluld is formed when photoexcited electrons, holes, and free excitons (TE) condense at low temperatures. In unstressed Ge the liquid phase consists of a fog of electron-hole drops (EHD) each several $\mu \mathrm{m}$ in size. (1) By sultably stressing a Ge crystal, a three-dimensional energy minimum for the EHL is formed in the intertor of the crystal. (2) In chls case, photoexc1ted carrlers, FE, and EHD are accelerated into this strain well and coalesce Into a single large electron-hole drop; the liquid is then a strain-confined electron-hole liquid (SCELL). The drop size varies with excitation leve ${ }^{(3,4)}$ and can be varied from $R \leq 50$ Hil up to the size of the well, $R \approx 700 \mu \mathrm{m}$ in some cases.

It has been found ${ }^{(5,6)}$ that in the SCEHL the electron-hole pair density varles with position. At first this is a surprising result, since a liquid is generally considered to have a constant density. However, the density variation in the SCEHL can be understoor ather simply ${ }^{(7)}$ by noting the following: the strain energy is parabolic ${ }^{(2)}$ with the distance from the center of the well, $E_{S} \propto \alpha r^{2}$; the chemical potential 15 uniform throughout the liquid volume; and the gas pressure outside the liquid and the surface pressure can both be neglected at low temperature. These assumptions give to first order ${ }^{(7)}$ a density distribution

$$
n(r)=n_{0}\left[1+Q\left(R^{2}-r^{2}\right)\right]
$$

Here $n(r)$ is the density at the position $r, R^{\prime}$ is the drop radius, $a=\alpha /\left(n_{0}^{2} E_{0}^{N}\right), E_{0}^{n}=\left.\frac{d^{2} E}{d n^{2}}\right|_{n_{0}}$, and $n_{0}$ is the equilibrium density. A more 
exact theoretical treatment ${ }^{(6)}$ gives a result similar to Eq. (1) with $\theta$ a function of $R$. Thus the SCEHL is compressed by the strain gradient, resulting in an increased density at the center of the drop which falls off to the equilibrium value at the surface. For relatively small drop size, $R \leq 150 \mu m$, the density variation is $\leqslant 20 \%$, depending on the parameter's of the experiment, particularly the strain parameter $\alpha$. For these small drop sizes, then, the equilibrium properties of the SCEHL can be studied. (3) For the largest drop sizes, $R \approx 700 \mu \mathrm{m}$, density 1ncreases of up to a factor of three have been observed by directly measuring the density profile. $(5,6)$ The agreement between experimental and theoretical density profiles is good.

At first, the density variation may seem like a complication in understanding simple experimental results such as the total luminescence decay time and lineshape vs excitation level, because the observed luminescence represents a superposition from liquid in all parts of the strain well. However, once the density varlations are understood, these experiments can be simulated theoretically, and different models can be tested. In fact, the changes in density with drop size can be utilized In studying the properties of the SCEHL over an exceptionally wide range of densities simply by varying the drop size, 1.e. the excitation level

$A$ second method to vary the density at a fixed temperature is to apply an external magnetic fleld. Experiments on unstressed Ge showed that the density oscillates with magnetic fleld as electron Landau levels pass through the Fermi level. ${ }^{(8)}$ For the strain-confined EHL, If a suffictently small drop size is chosen, a uniform density can be varied directly without the complications of compression. In this type of 
experiment density oscillations of $\sim 10 \%$ can be obtained for $H \leq 20 \mathrm{k} 0$.

In this paper we w111 discuss the dependence of the varlous recombination processes on density. The total recombination rate is assumed to vary wth density as follows:

$$
\begin{aligned}
\tau^{-1}(n) & =\tau_{d d}^{-1}+\tau_{\text {rad }}^{-1}(n)+\tau_{A n g}^{-1}(n) \\
& \approx A+B n+C n s .
\end{aligned}
$$

Here the first term represents processes Independent of denstry; the aecond term represents the usual radlatlve recombination processcs; and the third term represents Auger processes, where $s$ is expected to be 2 or 3. Efficiences for these processes can be defined as follows:

$$
\begin{aligned}
& \varepsilon_{d I}(n)=\tau(n) / \tau_{d i} \\
& \dot{r a d}_{\text {ad }}(n)=\tau(n) / \tau_{\text {rad }}(n) \\
& \epsilon_{\text {Aug }}(n)=\tau(n) / \tau_{\text {Aug }}(n)
\end{aligned}
$$

The experiments were performed on a $4 \times 4 \times 3 \mathrm{~mm}^{3}$ sample of ultra-pure, $N_{A} \leq 10^{21} \mathrm{~cm}^{-3}$, dislocation-free Ge grown by Hansen and Haller. (9) The sample was cut along crystallographic axes and etched in $311 \mathrm{NO}_{3}:$ IIF. The stress was applied from above along a (111) axis by a rounded nylon plunger in the Inhomogeneous geometry described in Kefs. (2) and (3).

The experimental setup was standard: a mechanically chopped ArgonIon laser or a pulsed GaAs laser was focussed onto a (110)-face of the Ge sample immersed in pumped liquid hellum within a superconducting solenold. Luminescence from the LA phonon replica at about 707 meV was collected from the opposite face, focussed onto the 
front slit of a spectrometer, and detected by a cooled high-sensitivity Ge photodiode. A signal averager or boxcar, was used to obtain data as a function of time with $10 \mu \mathrm{sec}$ resolution.

Two types of experiment will be discussed here. In the first experiment, the luminescence was recorded as a function of time after laser cutof for a series of different excitation levels. The luminescence was collected from the entire drop, without spatial or spectral selection. Because the average density Increases with drop size, the decay is non-exponential (see for example Ref. (3)), and therefore the initial decay time $\tau_{1}$ is of interest. In addition, in order to eliminate complications arising from variations in production efficlency with. power, the initial decay time was recorded as a function of initial drop size. The drop size was estimated as follows: the focussed luminescence image of the crystal was translated past a spectrometer slit, thus producing a spatial slit scan of the luminescence. The full width at half inaxlmum (FWtRI) of the slit scan is then used as an estlmate of the drop size. $(3,6)$

The results of this experiment are shown as the dots in Fig. 1, where the Inicial decay time $T_{1}$ of the total EHL luminescence 1 s plotted as a function of the inftial FWlM drop size, It should be noted that the equilibrium density and lifetime for this sample

$$
\begin{aligned}
& \mathrm{n}_{0} \approx 0.50 \times 10^{17} \mathrm{~cm}^{-3} \\
& \tau\left(n_{0}\right) \approx 500 \mu \mathrm{sec}
\end{aligned}
$$

are typical of the SCEHL in dislocation-free Ge. $(3,4,10,11,12)$

The curves in Fig. 1 represent calculations of the initlal luminescence decay time vs drop size, using calculated density distributions (6) 
which are in good agreement with the measured prof1les, (6) and using different values for the parameters in Eq. (2). The dot-dashed curve represents $\varepsilon_{d 1}=0 ., \varepsilon_{\text {rad }}\left(n_{0}\right)=0.75, \varepsilon_{A{ }_{B}}\left(n_{0}\right)=0.25$, and $s=2$. This model was formulated using experimental indications ${ }^{(3)}$ that

$$
\varepsilon_{\mathrm{rad}}(\mathrm{SCEHL}) \approx 3 \varepsilon_{\mathrm{rad}} \text { (unstressed EHL) }
$$

and the evidence obtained by Betzler et al. (8) that $A$ is negligible and that $\varepsilon_{\text {rad }} \approx 25 \%$ for unstressed Ge. Th1s model 1s clearly unsatisfactory; the Initial decay time decreases too much as the average density Increases, Indicating that the Auger term is too large. Thus an Auger process is not dominant here, as is belicved to be the case in unstressed Ge. ${ }^{(8,13)}$ The dashed curve represents $\varepsilon_{\mathrm{di}}=0 ., \varepsilon_{\mathrm{rad}}\left(\mathrm{n}_{0}\right)=1.0$, and $E_{\text {Aug }}\left(n_{0}\right)=0$. In this model, the change in $\tau_{1}^{-1}$ with drap size corresponds to the change in a kind of average density, as can be seen qualitatively from Eq. (2). This model is also unsatisfactory. The solid curve represents $\varepsilon_{d 1}=0.75, \varepsilon_{\mathrm{rad}}\left(n_{0}\right)=0.24, \varepsilon_{\mathrm{Aug}}\left(n_{0}\right)=0.01$, and $s=2$, which $1 \mathrm{~s}$ an approximate fit to the data. It is evident that a dominating density Independent recombination mechanism is necessary to explain this data. Additional evidence of the importance of the density independent recombination mechanism can be obtained from a second experiment. In this experiment the luminescence Intensity from the entire drop was monitored as a function of magnetic field at discrete times after a pulse from a GaAs laser. Under these condtctons the drop grows very rapidly (12) to a maximum size and then decays in the same way as a drop formed by cw excitation. The total energy per pulse was chosen to produce a drop with radius $R=125 \mu \mathrm{m}$. The magneto-oscillations of the luminescence at 
several delay times are shown in Fig. 2 , for $H \leq 18 \mathrm{kOe}$. It can be seen that at $t=I$ msec the oscillations are reduced in amplitude but have not changed sign, where $t=1 \mathrm{msec}$ is approximately twice $\tau\left(\mathrm{n}_{0}\right)$. Thts is in contrast to the case for unstressed $\mathrm{Ge}$, where the oscillations change sign after approximately $1 / 3$ of the zero-fteld decay $t$ ime.

To understand the significance of this result it is necessary to consider the kinetics of a pulsed experiment. Because the drop lifetime is much longer than the laser pulse width, $\approx 100$ nsec, the drop initially contains a constant number $N(t=0)$ of $e-h$ pairs, assuming tile pumping efficiency is independent of magnetic fleld. The lumiescence intensity as a function of time is given by

$$
I(H, t)=\frac{N(t)}{\tau_{\text {rad }}(n)}=B n N(0) e^{-t / \tau(n)}
$$

At a magnetic field for which the equilibrium density is higher than the zero field value $n_{0}$, the initial luminescence intensity is greater than $1(0,0)$ and the lifetime $\tau(n)$ is shorter than $\tau\left(n_{0}\right)$. Therefore at some later time $t_{T}$

$$
1\left(0, t_{T}\right)=1\left(H, t_{T}\right) ;
$$

at this turnover time the magneto-oscillations change sign. Assuming $n(H)-n_{0} \ll n_{0}$ a straightforward analysis yields

$$
t_{T}^{-1}=\mathrm{Bn}_{0}+\mathrm{sCn}_{0}^{\mathrm{s}}
$$

This result combined with equation (2) yields

$$
\frac{\tau\left(n_{0}\right)}{t_{T}}-c_{\text {rad }}\left(n_{0}\right)+\theta \varepsilon_{\text {Aug }}\left(n_{0}\right) \equiv \xi .
$$


The quantities $\xi$ and

$$
1-\xi=\varepsilon_{d 1}-(s-1) \varepsilon_{A u g}\left(n_{0}\right)
$$

appear throughout the analysis of magneto-osclllatory phenomena. Other combinations of recombination efflctencles cannot easidy be obtained from these experiments. If $\xi<1.0$, it can be seen from Eq. (10) that the density Independent recombination mechanism 1s more Important than the Auger mechan1sm. Conversely, if $\xi>1.0$, the Auger mechan 1 sm is more 1mportant than the density independent mechanism (for $s=2$ ). The data displayed In Fig. 2 give direct information about $\xi$. Since the turnover $t$ ime

$$
t_{T} 22 \pi\left(n_{0}\right),
$$

we conclude that

$$
E \leq 1 / 2
$$

In addition, from Eq. (10) we find that

$$
\varepsilon_{\mathrm{d} 1} \geq .50
$$

in agreement with the results of $\mathrm{FIg}_{\mathrm{g}}$. 1. We find from the data of Betzler et al ${ }^{(8)}$ that $\xi \sim 2$ for the EHL in unstressed Ge. This Indicates that the Auger mechanism dominates for this case.

Betzler et al. ${ }^{(8)}$ concluded that the density, independent recombination efficlency was less than $10 \%$ of the Auger efficiency and therefore set $\varepsilon_{\mathrm{d} 1}=0$. However, we note that if $\varepsilon_{\mathrm{d} i}=0.05$, and $\tau=36 \mu \mathrm{sec}$ for unstressed Ge this corresponds to a value of 


$$
\tau_{d 1}=\tau / \varepsilon_{d 1}=720 \mu \mathrm{sec} .
$$

For the strain-confined EHL, the same densfty Independent decay time corresponds to

$$
\varepsilon_{d 1}-\tau\left(n_{0}\right) / \tau_{d 1}=0.69 \text { (ULEHL) }
$$

using $\tau\left(n_{0}\right)=500 \mu \mathrm{sec}$. It 1s clear that such a process which can be neglected in unstressed Ge can at the same time dominate the decay time for the SCEHL.

We belleve that a density independent recumbination tim of scveral hundred usec is reasonable for Ge. Posstble reconbination sites include shallow traps and deep traps. A detailed calculation is beyond the scopi of this paper. However, crude estimates can be made of the required concentrations of shallow or deep recombination centers. First, we note that several groups $(14-16)$ have measured the EHL decay time in doped (unstressed) Ge. While there is some variation in the results, the lifetime generally starts to decrease when the impuricy concentration $N_{1}$ is greater than $\sim 10^{15}-10^{16} \mathrm{~cm}^{-3}$. For example, Zhurkin et al. (16) report $T=24 \mu \mathrm{sec}$ for $\mathrm{N}_{1}=2 \times 10^{26} \mathrm{~cm}^{-3}$ As impurities. If the e-h pair density remalns constant at these doping levels, the decrease in lifetime may be attributed to impurity-induced recombination. The change in lifetime then corresponds to $\tau_{\mathrm{d} f} \sim 70 \mu \mathrm{sec}$ for $\mathrm{N}_{1}=2 \times 10^{16} \mathrm{~cm}^{-3}$. Assuming further that this recombination rate is proportional to the impurity concentration, $\tau_{d 1}-700$ usec would correspond to $N_{1} \sim 2 \times 10^{15} \mathrm{~cm}^{-3}$ of shallow Impurtt1es. An estimate can also be made for deep levels, where nonradiative 
recotibination could take place via multiphonon emission. Experimental values for nonradlative capture crossmections at room temperature have been tabulated for $\mathrm{Ge}{ }^{(17)}$ and range from $\sigma_{\text {cap }} \sim 10^{-14}-10^{-16} \mathrm{~cm}^{2}$. A aimple model predicts that the capture rate $1 \mathrm{~s} \sim \sigma_{\text {cap }} \times v \times N_{1}$, where $v$ 1s the carrier velocity which can be taken to be the Fermi velocity for carriers in the EHl. In this case $\tau_{d 1}-1 \mathrm{msec}$ wauld correspond to $N_{1}-10^{10} \mathrm{~cm}^{-3}$. Our samples have not been fully charactertzed to this level, although it is known that the concentration of shallow Impurities 1s $\leq 10^{22} \mathrm{~cm}^{-3}$ and that levels are present assoctated with hydrogen. (18) Thus the study of the SCEIL In differently prepared samples may prove to be ansitive test of impurity-induced recombination.

\section{ACKNOWLEDGEMENTS}

We wish to acknowledge the actlve support of C.D. Jeffries. We thank E. E. Haller and W. I. hansen for provlding Ge samples and C. D. Jeffries, C. Kittel, L. Falicov, and E. E. Haller for Informative discussions. This work was supported by the Division of Materials Sciences, Office of Baslc'Energy Sclences, U. S. Department of Energy. 


\section{REFERLNCES}

1. For comprehensive reviews see (a) experfment al: J.C. Hensel, T.G. Phlllips, and G.A. Thomas, Solid State Phys. 32, Henry Ehrenrefch, Frederick Seltz and David Turniull ed., 88 (Academic Press, 1977); (b) theoretical: T.M. Rice, Ibld., 1.

2. R.S. Markiewicz, P. Holfe, and C.D. Jeffries, Phys, Rev. B 15, 1988 (1977).

3. J.P. Wolfe, R.S. Marklewicz, S.M. Kelso, J.E. Furneaux, and C.D. Jeffries, Phys. Kev. B, to be published.

4. C.D. Jeffries, J.P. Wolfe, S.M. Kelso, R.S. Markiewicz, and J.E. Furneaux, J. Lum. 12/13, 659 (1976).

5. S.M. Kelso, R.S. Marklewicz, and J.E. Furneaux, Bull. Am. Phys. Soc. 22, 269 (1977).

6. S.M. Kelso, et al. to be published.

7. R.S. Markiewicz and S.M. Kelso, Solid Stace Commun. 25, 275 (1978).

8. K. Betzler, B.G. Zlurkin, A.L. Karuzskii, and B.M. Balter, J. Lum. 12, 651 (1976); K. Betzler, B.G. Zhurkfn, and A.L. Karuzsk11, Solid State Commun. 17, 577 (1975); A.L. Karuzsk11, K. Betzler, B.G. Zhurkin, and B.M. Balter, Pis'ma Zh Eksp. Teor. Fiz. 22, 65 (1975) [JETP Lett. 22, $29(1: 75)]$.

9. W.L. Hansen and E.E. Haller, IELE Trans. Nucl. Sc1. 21, 251 (1974). 10. Ya. E. Pokrovski1 and K.I. Svistunova, 2h. Eksp. Teor. F1z. Pis'ma Red. 23, 110 (1976) [JETP Lett. 23, 95 (1976)].

11. J.C.V. Mattos, J.M. Worlock, and T.C. Damen, Solid State Commun. 22, 13 (1977). In this experiment a dislocated sample was used, resulting In a reduced iffetime. 
12. J.E. Furneaux, J.P. Wolfe, and C.D. Jeffries, Soldd State Commun. 20, 317 (1976).

13. C. Benốt à la Gulllaume, M. Voos, and F. Salvan, Phys. Rev. Lett. 27, 1214 (1971); R.F. Leheny, J. Shah, and M. Voos, Soldd State Commun. 20, 819 (1976).

14. C. Benô̂t à la Gulllaume, M. Voos, Solld state Conusun. 11, 1585 (1972).

15. A.L. Karuzsk11, K. Fotzler, B.G. Zhurkin, V.P. Aksenov, Fiz. Tverd. Tela. 17, 3104 (1975) [Sov. Phys. - Sol1d State 17, 2058 (1975)].

16. B.G. Zhurkin, A.L. Karuzsk11, R.L. Korchazlikina, V.A. Fradkov, Solid State Commun. 20, 341 (1976).

17. E. P. Sinyavski1 and V. A. Kovarskii, Fiz. Tverd. Tela. 9, 1464 (1967) [Soviet Physics - Solid State g, 1142 (1967)].

18. E. E. llaller, private communication. 


\section{FI"NE CAPTIONS}

F1g. 1. Intial decay time $\tau_{1}$ for the total luminescence intensity at $\approx 707 \mathrm{meV}$ plotted vs drop size estimated by the full width at half maximum (Finy) of a s] it scan, for the strain-confined electron-hole liquid (SCEHL) in Ge. Sample CR50 was inhomogeneously stressed along a $(111)$ axis. $T=1.9 \mathrm{~K}$. The dots are the experimental results; the three curves are calculations discussed in the text.

Fig. 2. Total luminescence intensity as a function of magnetic ficld $\mathrm{u}$ for several different delay tiries after an excitation pulse, for the same sample as in Fig. 1. H (10̈0), T = $1.6 \mathrm{~K}$. The vertical scale is of fset from zero by an arbitrary amount. The symbols at the right indicate $1.0 \%$ of the total intensity. 


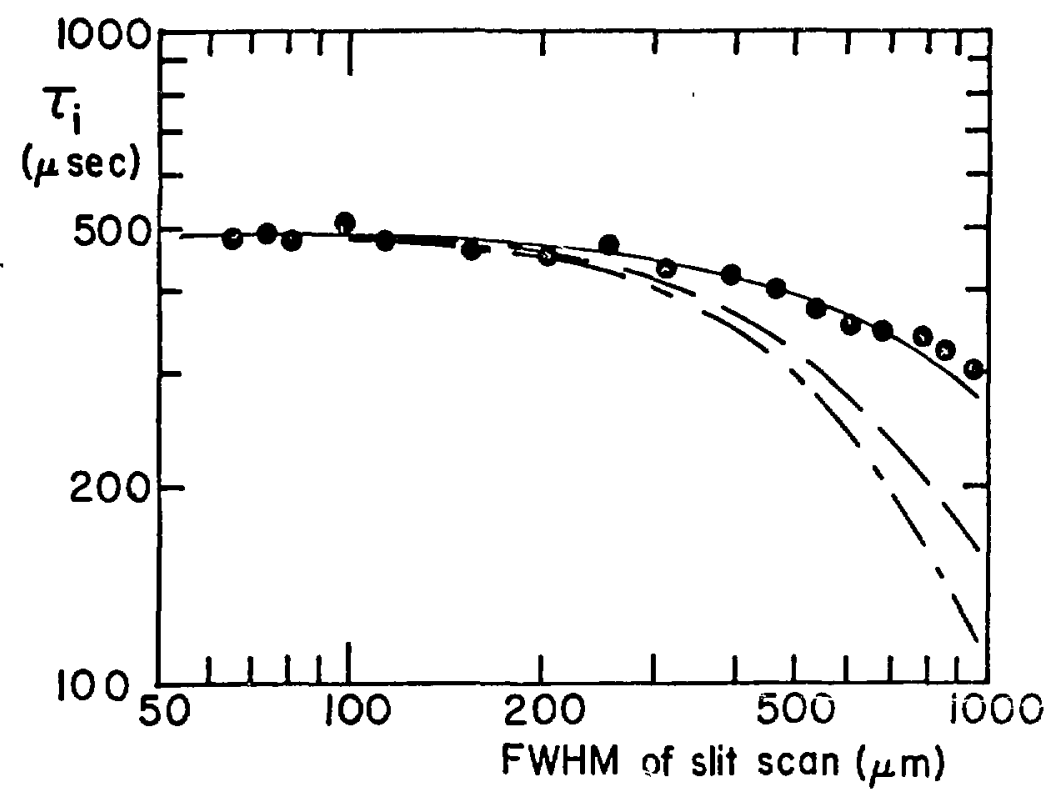

Fig. 1 


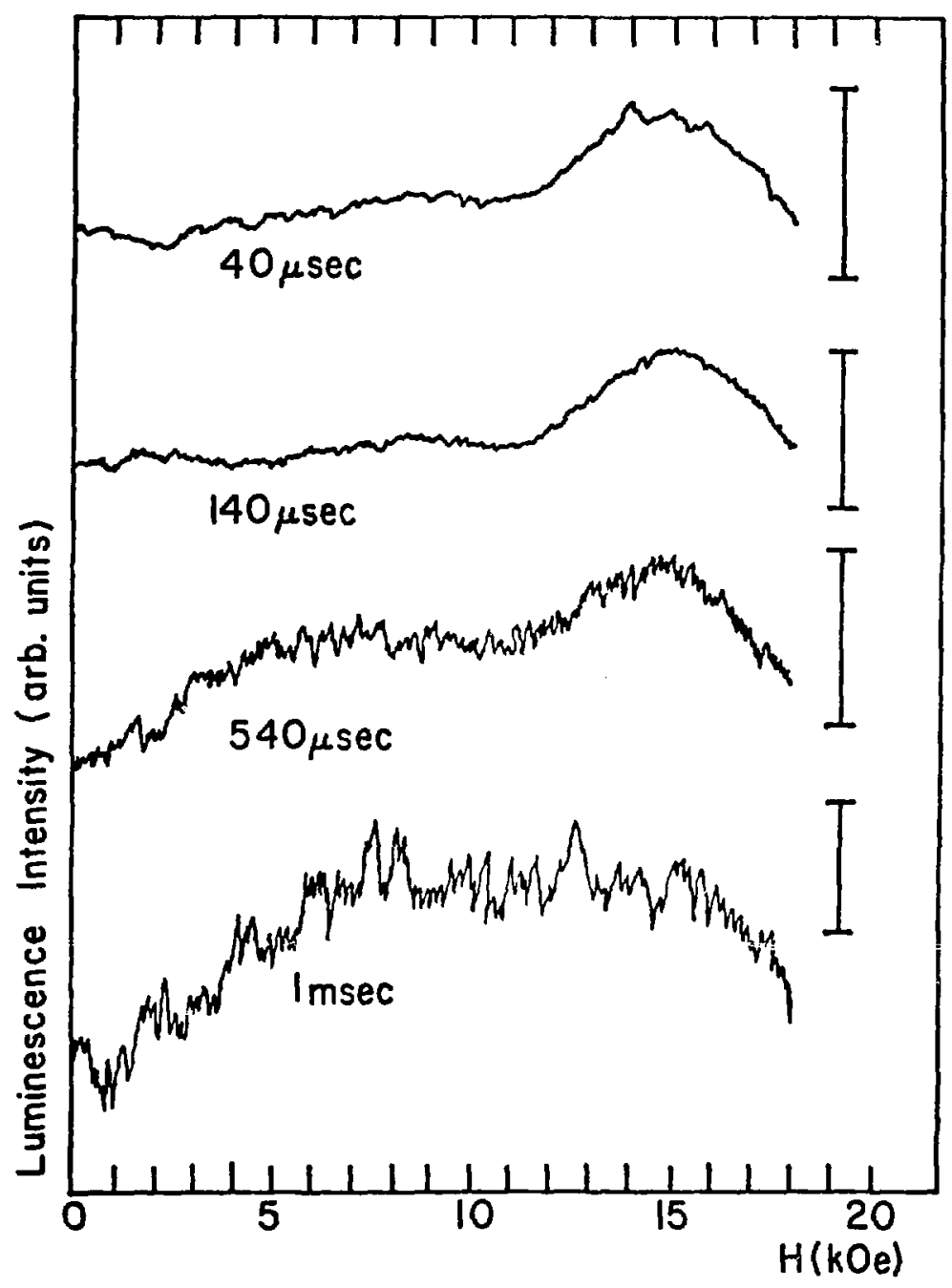

Fig. 2 